\title{
NUMERICAL INVESTIGATION OF ROD-AIRFOIL CONFIGURATION AEROACOUSTIC CHARACTERISTICS USING FFOWCS-WILLIAMS-HAWKINGS EQUATIONS
}

\author{
Ece Ayli ${ }^{1}$, Eyup Kocak ${ }^{2}$, Hasmet Turkoğlu³
}

\begin{abstract}
The rod-airfoil configuration is a fundamental study to understand sound generation processes and the acoustic phenomena in the application of turbines, fans, and airfoils. In the present research, the noise that is originated by the rod-airfoil configuration is examined using numerical methods which are Large Eddy Simulation (LES), and Reynolds Averaged Navier Stokes (RANS) models, coupled with an FFOWCS-WILLIAMSHAWKINGS (FW-H) technique. For the RANS method, k- $\omega$ SST and Spalart Allmaras (S-A) turbulence models are utilized in order to investigate the capability of different models for the analysis of the aeroacoustic flow field. The ANSYS FLUENT solver is chosen to carry out the numerical simulations. The examined rod and chord diameter Reynolds numbers are 48000 and 480000 , respectively and the Mach number is 0.2 . Results are obtained for both in the near field and acoustic far-field. The obtained numerical results are verified with an experimental study from the literature, and the results of both approaches are compared with each other and the experiment. Comparisons are performed for mean velocity profiles in the rod and airfoil wakes, pressure spectra and power spectral density. The results obtained show that LES is preferable for this problem as it is capable of capturing the flow separation, reattachments, vortex street, and various length scales of turbulence. Although both RANS and LES methods provide a consistent flow field with experimental methods, the RANS approach overestimates the vortex shedding frequency and Strouhal number. The RANS model predicts the flow field well; however, it overestimates the noise spectra. The LES model predicts satisfactory acoustic spectra.
\end{abstract}

Keywords: Large Eddy Simulation (LES), FW-H Technique, Computational Fluid Dynamics, Multi-zone mesh, Noise Reduction, Noise Generation

\section{INTRODUCTION}

The fast development of industry and economy created a demand in the cargo and passenger transportation systems. To meet this demand, the civil aviation industry has developed rapidly and has an essential role in the global economy. In time, like all of the other technological areas, the associated negative environmental efficacy of aviation, including both air pollution and noise problem, has been attracting attention. The aircraft noise mechanism is very complex, which comprises strong interactions between incoming turbulent flow and airfoil blade. To predict this interaction and understand the flow and the noise phenomena, hybrid methods, which are the combination of the Computational Fluid Dynamics (CFD) solvers and Lighthill's analogy, have been used. Using the hybrid method for the rod-airfoil test cases, the broadband noise has been adequately modeled in recent studies [1-3]. Therefore, the rod-airfoil configuration has been considered as a benchmark for sound generation processes in turbomachines and airfoils.

Casolino et al. [1] are one of the first researchers who used the unsteady two-dimensional RANS method to analyze this problem. They used a statistical model coupled with FW-H [2,3] analogy to model the three-dimensional

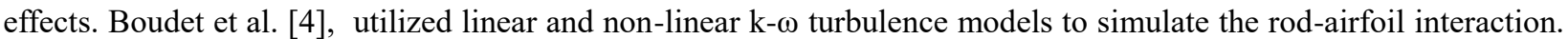
They showed that the two-dimensional RANS approach is an efficient tool for the simulation of rod-airfoil interaction.

This paper was recommended for publication in revised form by Regional Editor Muslum Arici

${ }^{1}$ Department of Mechanical Engineering, Cankaya University, Ankara, Turkey

${ }^{2}$ Department of Mechanical Engineering, Cankaya University, Ankara, Turkey

${ }^{3}$ Department of Mechanical Engineering, Cankaya University, Ankara, Turkey

*E-mail address: eayli@cankaya.edu.tr, eyupkocak@cankaya.edu.tr, hasmet@cankaya.edu.tr, Orcid id: /0000-0002-6209-161X, 10000-0002-1544-2579, 0000-0002-1941-986X

Manuscript Received 14 January 2020, Accepted 06 March 2020 
Large Eddy Simulation (LES) method has also been used greatly to study this problem. Lafon et al. [5] used the LES method to simulate rod-airfoil flow configuration, and they compared their results with experimental data of Jacob et al. [6]. It was pointed out that the aerodynamic and acoustic behaviors are well predicted using high-order discretization tools on an overlapping topological grid. There was a good agreement between numerical and experimental results Eltaweel and Wang [7] used an incompressible LES solver for the same problem. Acoustic spectra are computed using the Lighthill's analogy, and their results show fair agreement with the experimental data for both velocity statistics and energy spectra. Agrawal and Sharma [8], used two different LES solvers.

The first method solves the compressible flow equations by using FW-H analogy (Charles) and the second method is PisoFoam that solves the incompressible flow equations using Amiet's theory. According to their results, both methods have similar mean flow distribution, and satisfactory results according to the comparison of experimental data.

Insight of the recent studies, the LES model proves itself that it is a capable tool to model and predict the farfield noise. Several numerical studies that use LES show a satisfactory agreement with the experimental researches. As shown by the Greschner et al. [9], Detached Eddy Simulation (DES) turbulence models are an excellent competitor for this kind of problem with less computational costs when it is compared with LES. DES model which is proposed by Spalart et al. [10], is a hybrid model that uses RANS in the near-wall and uses LES inflow zones. However, DES is still undergoing improvements and needs more benchmarking and comparison with RANS, LES and experimental studies. In another study, Greshner et al. [9] compared the standard k- $\varepsilon$ DES model with a hybrid DES/FW-H aeroacoustic model. They claimed that the new model that they propose is much more realistic than the standard model, where unsteady flow predictions are improved. Also, their hybrid models' accuracy reaches to the LES model. Samion and Ali [11] compare the LES and DES model for rod-airfoil noise calculation. According to their results, LES overpredicts the overall sound pressure than DES when they are compared with experimental results. When aerodynamic performance is examined, it is seen that LES provides better performance with catching vortex core more accurately. Galdeano et al. [12] used the DES model with Spalart-Allmaras in the RANS zone. Their results are in good agreement with the experimental results of Jacob et al. [6]. The detailed mechanism and physics of the rod -airfoil noise generation is still investigated. Caraeni et al [13], investigated the sound generated by rod-airfoil configuration numerically with a Detached-Eddy-Simulation (DES) approach. The acoustic levels are captured well with DES approach when it is compared with the experimental case. Researchers claimed that pressure based solver gives a good prediction of the overall broadband spectrum. Michel et al [14], also used DES methods in aeroacoustic applications. They calimed that The grid must be fine enough to resolve the sources and the propagation between the sources and the data surface. Greschner et al [15] utilized LES method to model broadband noise sources in rod-airfoil configuration. An implicit formulation is used with second order accuracy and a grid of 2.3 million cells. Laouria et al [16] used to ANSYS Fluent program to investigate the flow in cavity and they claimed that ANSYS is a powerful tool and obtained numerical results are coherent with the experimental results.

In the present article, for the rod-airfoil benchmark problem, RANS and LES methods coupled with an FW-H technique are used for the simulations, and the results are compared with each other and with the experimental study of Jacob et al. [6]. Results are obtained for both in the near field and acoustic far-field. Near- field data is obtained on the rod and airfoil surface with using FW-H analogy. Comparisons are made for both aerodynamic and aeroacoustic behavior of the rod-airfoil. Also, in order to find the dominant acoustic source, the acoustic field for flow over rod is investigated.

\section{MATHEMATICAL MODELS AND NUMERICAL SOLUTION Mathematical Model}

The schematic view of the rod-airfoil structure considered is shown in Fig.1a. Around the rod, eddies form and break. Hence, the flow structure is very complex; it is unsteady, chaotic, turbulent flow comprising vortices and separations.

The most accurate method is Direct Numerical Simulation (DNS), which aims to capture all eddy sizes including the smallest turbulent scale by solving Navier-Stokes equations directly. However, DNS requires very fine meshes and time step, and hence the computational cost is high. At this point, the LES model is an alternative 
approach with less computational effort and mesh resolution. In this approach, large eddies are computed directly while small scale motions are modeled [21]. Therefore, the accuracy of this model lies between DNS and RANS methods.

In the RANS approach, the averaged quantities and the effects of the instantaneous turbulent motion are predicted. The turbulence is modeled using models like k- $\omega, \mathrm{k}-\varepsilon$, and Spallart-Allmaras. For incompressible flows, the fundamental equations are given as follow in Eq. (1) and Eq. (2).

$$
\begin{gathered}
\frac{\partial \bar{u}_{i}}{\partial x_{i}}=0 \\
\frac{\partial \bar{u}_{i}}{\partial t}+\frac{\partial}{\left(\partial x_{j}\right)}\left(\bar{u}_{i} \bar{u}_{j}\right)=-\frac{1}{\rho} \frac{\partial \bar{p}}{\partial x_{j}}+\frac{\partial}{\partial x_{j}}\left(\frac{\mu}{\rho} \sigma_{i j}\right)-\frac{\partial \tau_{i j}}{\partial x_{j}}
\end{gathered}
$$

In Eq (2), $\tau_{-}$ij denotes the subgrid-scale stress and defined by Eq. (3).

$$
\tau_{i j}=\overline{\rho u_{i} u_{j}}-\bar{\rho} \bar{u}_{i} \bar{u}_{j}
$$

The subgrid-scale models are used in modelling the small scale turbulence while large scale turbulent flow is solved directly. In ANSYS Fluent solver, an eddy viscosity approach is used that relates the subgrid-scale stresses to the large-scale strain rate tensor $\left(\bar{S}_{i j}\right)$ with the following equation.

$$
\tau_{i j}-\frac{1}{3} \tau_{k k} \delta_{i j}=-2 \mu_{t} \bar{S}_{i j}
$$

In the RANS model, eddy viscosity represents all turbulent scales, but in the LES model, only subgrid-scale viscosity is modeled [22]. In the RANS method, Navier-Stokes equations are decomposed into the mean and fluctuating parts. Assuming the flow is incompressible, Reynolds averaged continuity and momentum equations are given as:

$$
\begin{gathered}
\frac{\partial \bar{u}_{i}}{\partial x_{i}}=0 \\
\frac{\partial}{\partial t}\left(\partial \bar{u}_{\imath}\right)+\frac{\partial}{\partial x_{j}}\left(\overline{u_{\imath} u_{j}}\right)=-\frac{1}{\rho} \frac{\partial \bar{p}}{\partial x_{i}}+\frac{\partial}{\partial x_{j}}\left[v\left(\frac{\partial \overline{u_{l}}}{\partial x_{j}}+\frac{\partial \overline{u_{j}}}{\partial x_{i}}\right)\right]+\frac{\partial}{\partial x_{j}} \overline{\left(u_{i}^{\prime} u_{j}^{\prime}\right)}
\end{gathered}
$$

In Eq. (6), $\overline{\left(u_{i}{ }^{\prime} u_{j}{ }^{\prime}\right)}$ term denotes the Reynolds stresses and needs to be modeled with a turbulence model. To relate the Reynolds stress term to velocity gradients, Boussinesq approximation is used. For this problem, twoequation k- $\omega$ SST and one equation S-A turbulence models are used to model the Reynolds stress term.

Spalart-Allmaras model is widely used in the aeronautics applications, which solves a modeled transport equation for the eddy turbulent viscosity. The turbulence viscosity is computed as it is given in Eq (7).

$$
\mu_{t}=\rho \tilde{v} f_{v 1}
$$

In Eq. (7), $f_{v 1}$ denotes the viscous damping function and given by

$$
f_{v 1}=\frac{X^{3}}{X^{3}+C_{v 1}^{3}}
$$


where $\mathrm{C}_{\mathrm{v} 1}$ is constant [14].

Two equation $\mathrm{k}-\omega$ turbulence model is based on transport equations for the turbulence kinetic energy and the specific dissipation rate. In the SST based k- $\omega$ model, in the free stream, k- $\varepsilon$ turbulence model and near the walls $k-\omega$ turbulence model are used by combining the advantages of both methods. Eddy-viscosity is computed as it is given by Eq. (9).

$$
\mu_{t}=\frac{\rho k}{\omega} \frac{1}{\max \left[\frac{1}{\alpha^{*}}, \frac{S F_{2}}{a_{1} \omega}\right]}
$$

where $\mathrm{S}$ is the strain rate and $\alpha^{*}$ is the turbulent viscosity causing a low-Reynolds number correction [18].

To predict aeroacoustics behavior of the flow; first, a time-accurate flow is predicted from the time-dependent behavior of the pressure on the selected locations (source surface). In the second step, the sound pressure signals are computed using the pressure data that are collected in the first step.

For the determination of sound pressure levels, FW-H integral method is used [19]. FW-H equation is given in $\mathrm{Eq}(10)$.

$$
\left(\frac{\partial^{2}}{\partial t^{2}}-c_{0}^{2} \frac{\partial^{2}}{\partial x_{i} \partial x_{i}}\right)\left(H(f) \rho^{\prime}\right)=\frac{\partial^{2}}{\partial x_{i} \partial x_{i}}\left(T_{i j} H(f)\right)-\frac{\partial}{\partial x_{i}}\left(F_{i} \delta(f)\right)+\frac{\partial}{\partial t}(Q \delta(f))
$$

where $\mathrm{t}, T_{\mathrm{ij}}, \mathrm{Q}$, and $\mathrm{f}$ represent time, Lighthill's stress tensor, unsteady force and unsteady mass, respectively. Function $\mathrm{F}$ denotes the domain outside the source surface.

\section{Numerical Solution}

There are several critical issues for solving the problems with a sufficient degree of accuracy and capture the flow phenomena in all scales in the spectrum. Firstly, the computational grid should be fine enough to resolve the Kolmogorov scale. Secondly, numerical methods should be chosen appropriately for each of the problems like the turbulence model, time step size, convergence criteria, and discretization method.

To solve the flow field and the sound pressure level, ANSYS FLUENT software is utilized. As a preliminary study, mesh independency study is performed. As shown in Fig. 1(b) and 1(c), to form the computational mesh system, the flow domain is divided into 14 zones. The mesh system is generated using ANSYS Meshing software with quadrilateral elements. The mesh is refined in the zone close to the rod and the airfoil in order to capture the vortex shedding well. For rod, airfoil and the area between the rod-airfoil zone $\Delta \mathrm{x}$ is $25 \times 10^{-5} \mathrm{~m}$ where the mesh is finer compared with other regions. In order to resolve the flow field accurately, the first grid next to solid surfaces has $y+<1.5$. Since LES approach requires finer grid resolution to resolve the large eddies, the mesh independency study is performed with the LES approach. The mesh independency study is carried out with four different quadrilateral mesh structures of $5 \times 10^{5}, 2 \times 10^{6}, 4 \times 10^{6}, 6 \times 10^{6}$ elements. For the mesh independency study, Strouhal number is chosen as a control parameter. In Figure 2, variation of Strouhal number with number of mesh elements is shown. As seen in this figure, the variation of the Strouhal number is very small for the mesh number of $3 \times 10^{6}$ and greater. $3 \times 10^{6}$ element grid provides satisfactory results with a maximum deviation of 0.088 compared to $4 \times 10^{6}$ element grid. Therefore, 3000000 number of element mesh is used in the numerical studies. 
Journal of Thermal Engineering, Research Article, Vol. 7, No. 2, Special Issue 13, pp. 58-70, February, 2021

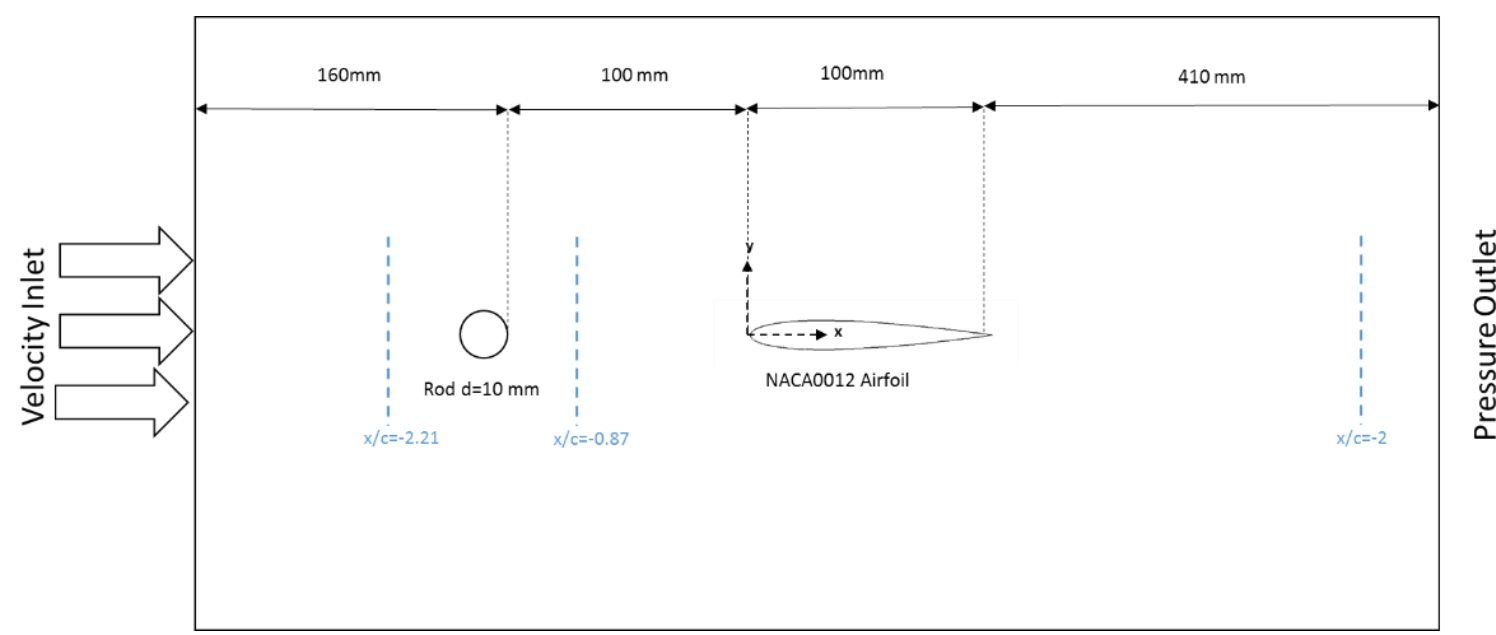

(a)

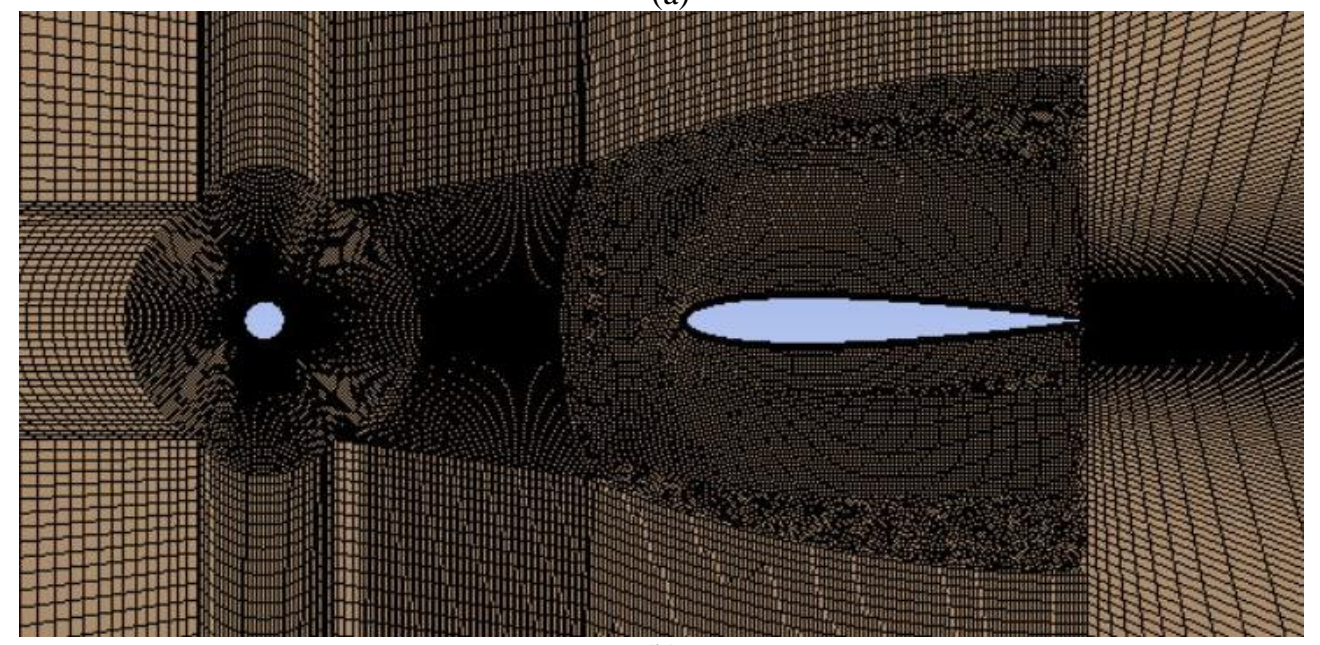

(b)

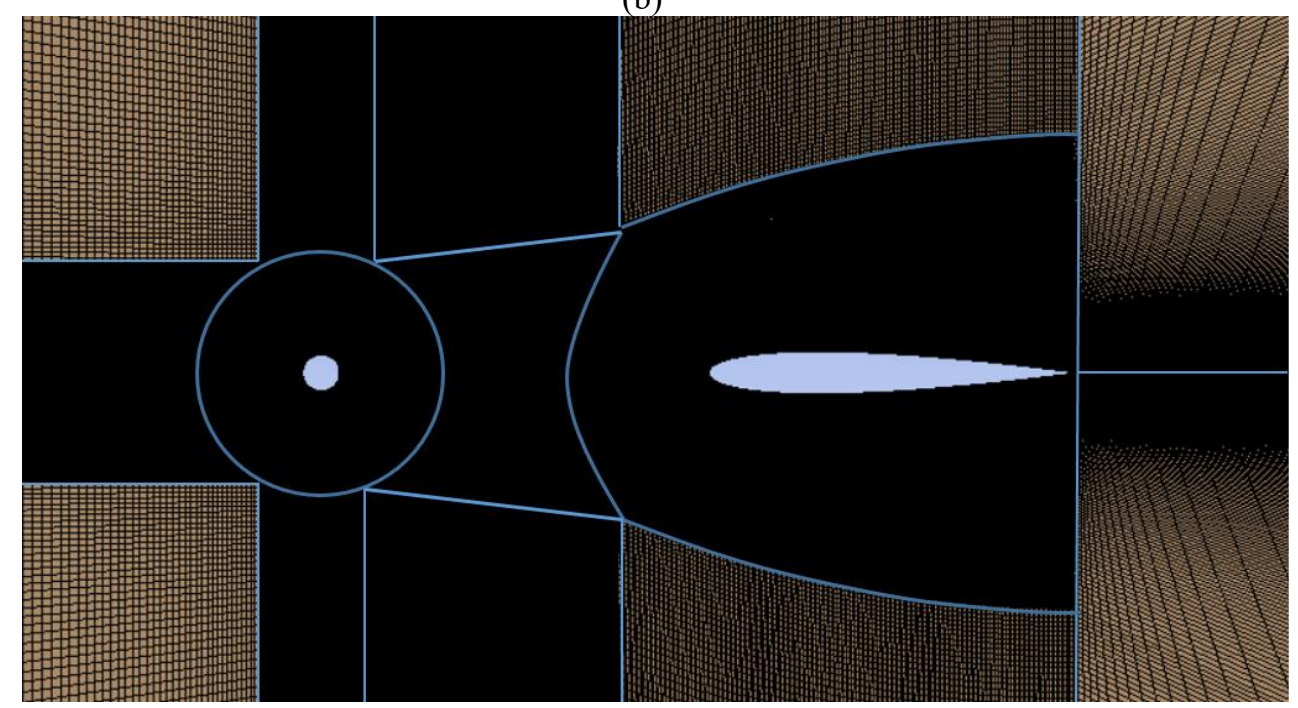

(c)

Figure 1. (a) Two Dimensional schematical view of the rod-airfoil configuration and boundary conditions (b) Computational grid system for rod-airfoil configuration with coarse mesh (c) with fine mesh 


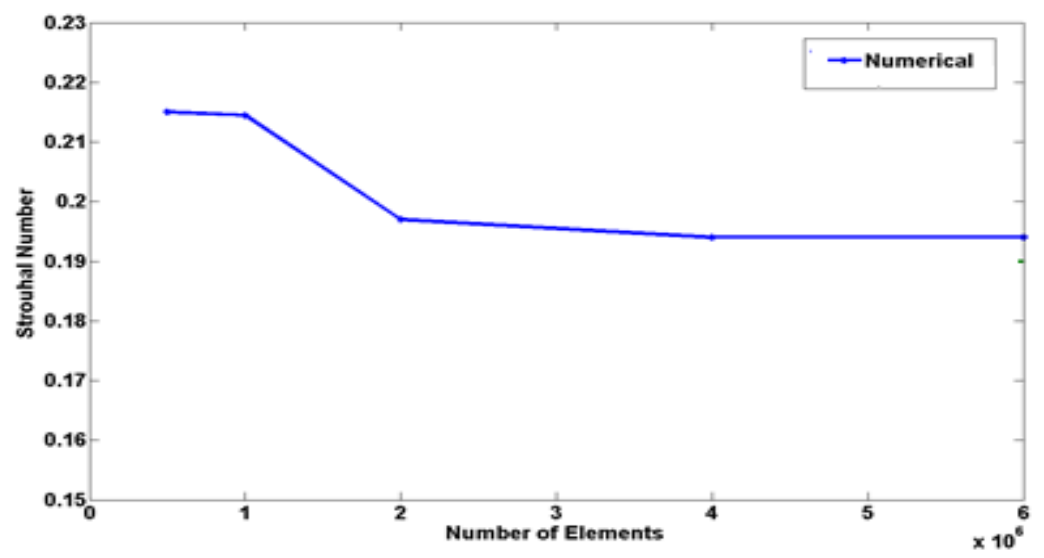

Figure 2. Variation of Strouhal number with the mesh number

\section{RESULTS AND DISCUSSION \\ Aerodynamic Results}

To check the accuracy of the numerical study, the results of numerical solutions are compared with the experiment of Jacob et al. as shown in Figure 3-6 [6]. The experimental configuration comprises NACA0012 profile and a rod with a $10 \mathrm{~mm}$ diameter. In the experiments, $\mathrm{u}_{\infty}$ is taken as $72 \mathrm{~m} / \mathrm{s}$. The examined rod and chord diameter Reynolds numbers are 48000 and 480000 , respectively and the Mach number is 0.2 . For the verification of the numerical methodology, computations are carried out for the same geometrical and flow conditions. In Figure (1(a)) schematical view of the flow domain and boundary conditions are given. Velocity inlet and pressure outlet conditions are used in the inlet and outlet of the domain, respectively. No-slip condition is applied at the rod and airfoil surfaces. The simulations are performed with a time step of $\Delta \mathrm{t}=5 \times 10^{-6} \mathrm{~s}$. The total time step considered is 100000 which corresponds to the physical time of $\mathrm{t}=0.5 \mathrm{~s}$.

In Figure 3, velocity distribution is obtained with k- $\omega$ SST turbulence model for 2-dimensional and 3dimensional geometry. It is observed that trends are almost same and 2-D simulations confirm with the experimental findings. Therefore, in order to minimize the computational costs, 2-D geometry is used in simulations.

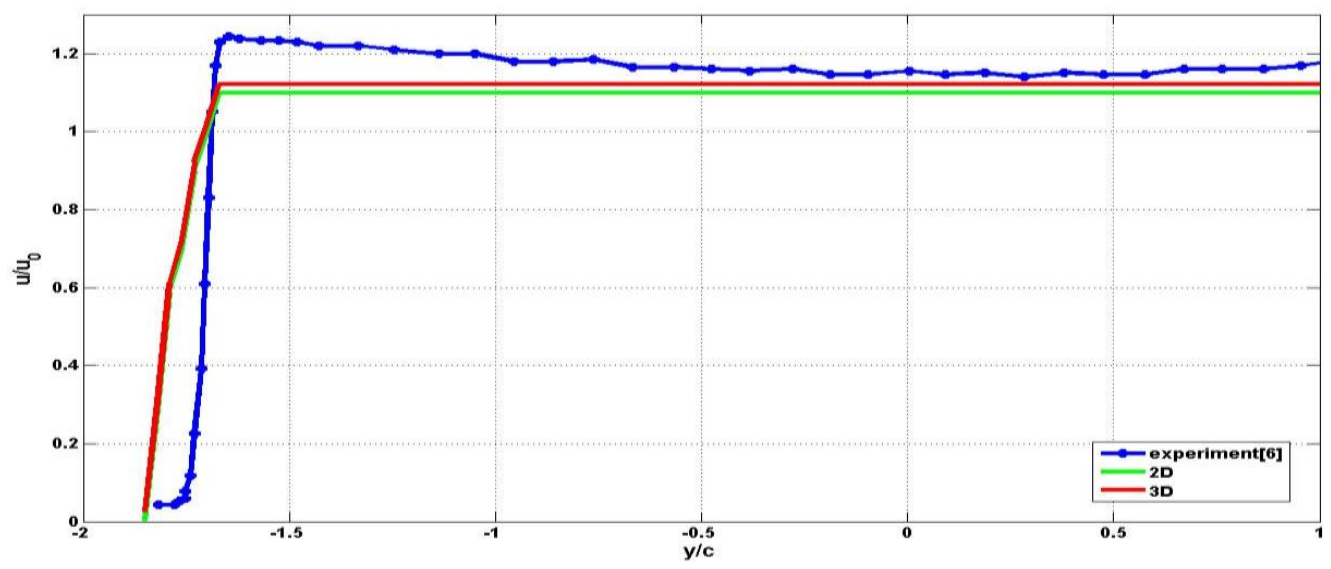

Figure 3. Comparison of $2 \mathrm{D}$ and $3 \mathrm{D}$ velocity distribution

A whole view of the flow field is presented in Fig. 4 with velocity contour obtained by RANS simulation. As seen in this figure, the rod causes shedding, and the vortices impinge on the leading of the airfoil. At the trailing edge of the airfoil, vortex structures rise again with distortion of the vorticity field. 


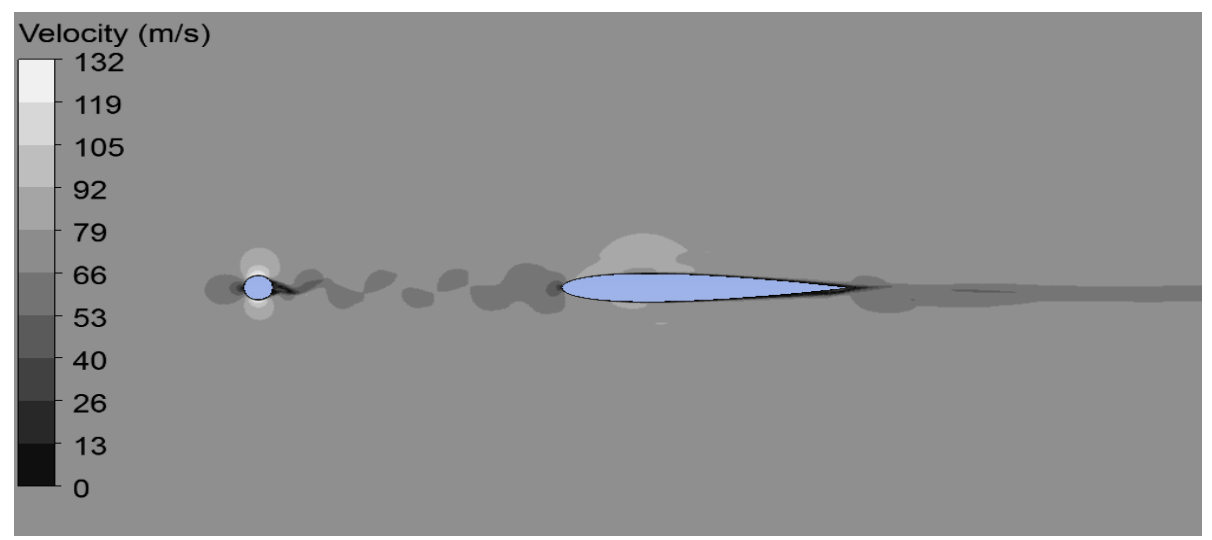

Figure 4. Velocity field contour of whole rod-airfoil structure.

To compare the results of the turbulence models used in the simulations, vorticity contours in the zone right before the leading edge of the airfoil is shown in Fig. 5. In this figure, experimentally obtained vorticity field and vorticity fields obtained by RANS and LES approaches are given. When these vorticity fields are analysed, it is seen that with LES approach, flow separation, vortex structures, and reattachment are captured better. The possible reason may be that the large eddies comprise most of the turbulent kinetic energy, which is solved directly by the LES model. With both approaches, counter-rotating vortices are well predicted; however, the shear layer transition is not predicted with the RANS method. With the RANS method, vortices are aligned in the rod axis and secondary vortices are not visible. In the LES approach, vortices disperse in the area between rod-airfoil in a random pattern which is also observed similarly in the experiment of Jacob et al. [6] (Figure 4). Also, Boudet et al.[4] observed the same phenomena with RANS and LES methods

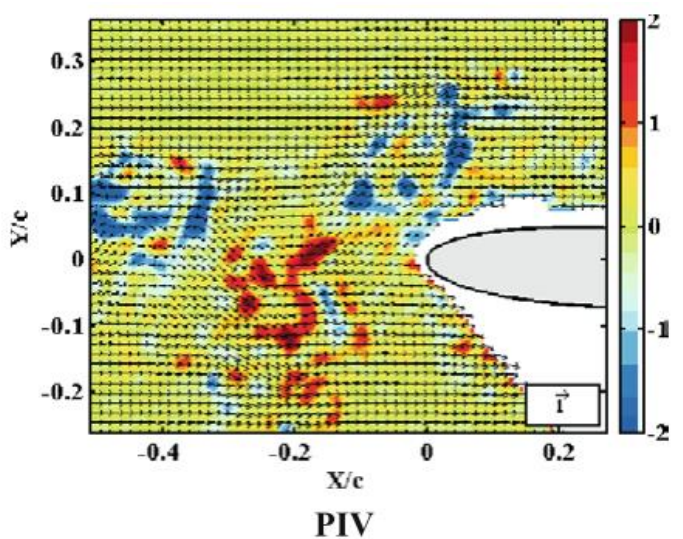

(a)

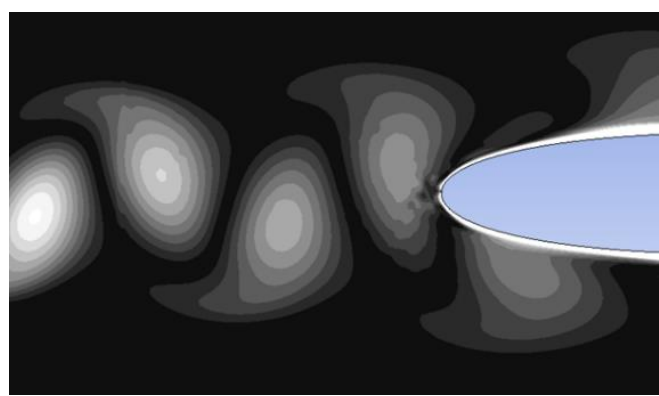

(b)

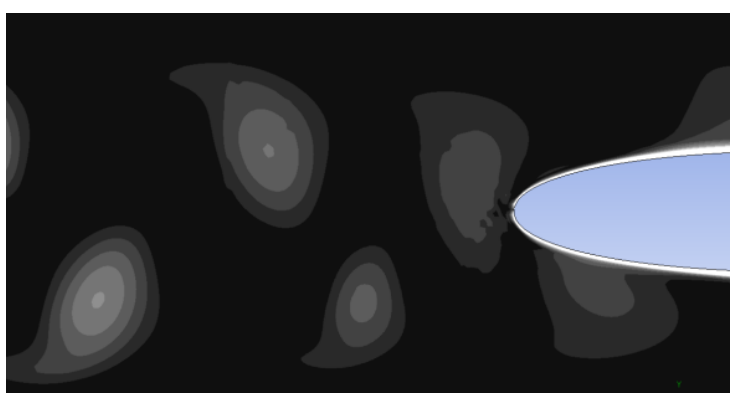

(c)

Figure 5. Vorticity from PIV [adopted from Jacob et al.,6 ](b) vorticity contour for RANS method (c) vorticity contour for LES method 
In Figure 6, the mean velocities at four different vertical planes at four different $\mathrm{x}$ positions are shown for experimental and numerical studies. The positions of the locations are indicated in Figure (1a). As seen in this figure, the trend of the variation of the velocity at $\mathrm{x} / \mathrm{c}=-2.21$ matches reasonably well with experimental results. The non-dimensionalized velocity profile between rod and airfoil is shown in Figure (5b). While in the free stream RANS models over predict the velocity, in the vortex shedding region RANS models underpredict the velocity distribution. When Figure 4 and Figure (5b) are examined together, it is pointed-out that shed vortices are caught better with the LES model than RANS models. Therefore, velocity distribution in the vortex street is predicted better with the LES approach. The calculated velocity profiles for the airfoil trailing edge $(\mathrm{x} / \mathrm{c}=2)$ is depicted in Figure $(5 \mathrm{c})$. With all the approaches used, the low-velocity zone is qualitatively well captured. As a partial conclusion, both of the methods predict the flow field fairly well. However, the LES model captures the velocity distribution better than the RANS model quantitatively.

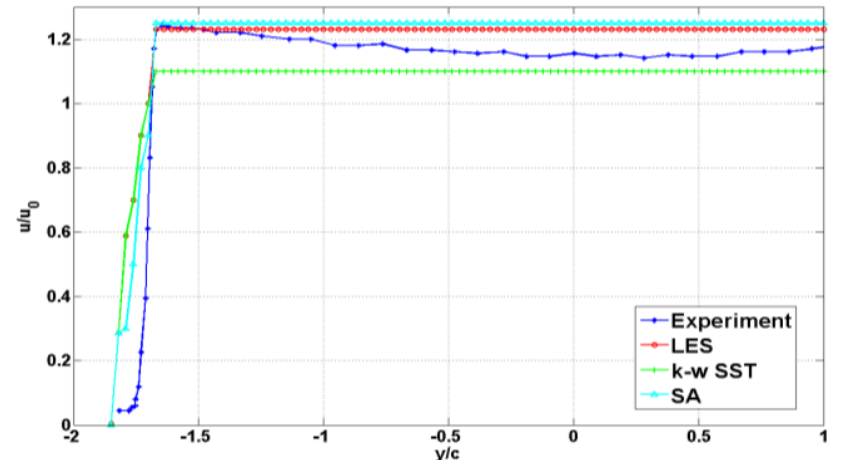

(a)

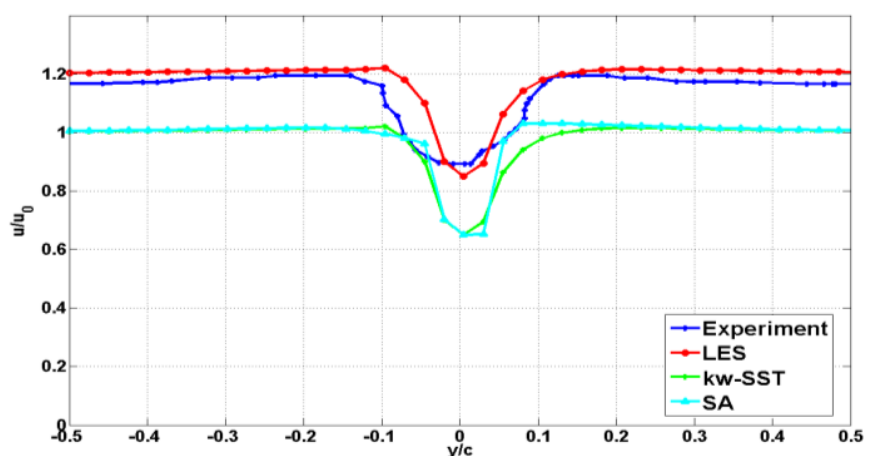

(b)

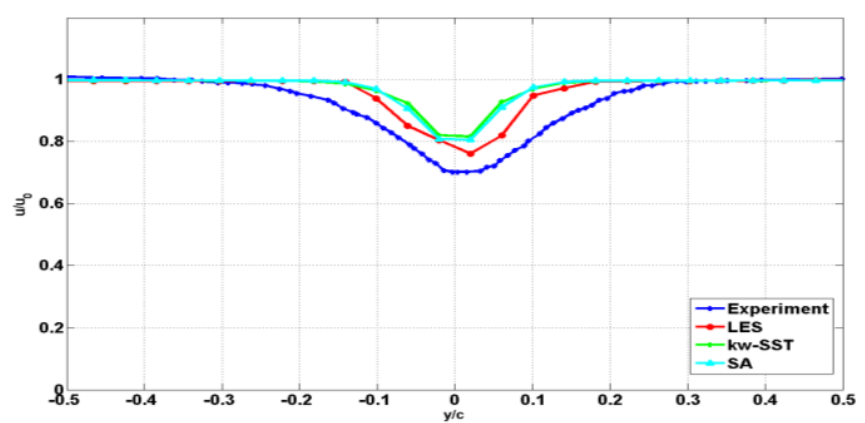

(c)

Figure 6. Mean velocity distribution for RANS, LES and experimental methods (a) incoming flow $(\mathrm{x} / \mathrm{c}=-2.21)$ (b) behind the $\operatorname{rod}(\mathrm{x} / \mathrm{c}=-0.87)(\mathrm{c})$ airfoil trailing edge $(\mathrm{x} / \mathrm{c}=2)$

In Figure 7, velocity contours for rod-airfoil configuration are depicted for S-A and k- $\omega$ SST turbulence model. With both models, vortex dispersion and interaction with the leading edge of the airfoil is observed. After the interaction with the leading edge, vortices are disrupted along the airfoil surfaces. In the leading edge of the airfoil, the mean velocity value in the vortex street is $15.5 \mathrm{u}_{\infty} \%$ and $14 \mathrm{u}_{\infty} \%$ for S-A and $\mathrm{k}-\omega \mathrm{SST}$, respectively. In the trailing edge, mean velocity values are decreases to $7.5 \mathrm{u}_{\infty} \%$ and $6.1 \mathrm{u}_{\infty} \%$ for S-A and $\mathrm{k}-\omega \mathrm{SST}$, respectively. Stagnation point in the leading of the airfoil and pressure blobs in the airfoil sides are observed with both approaches. 


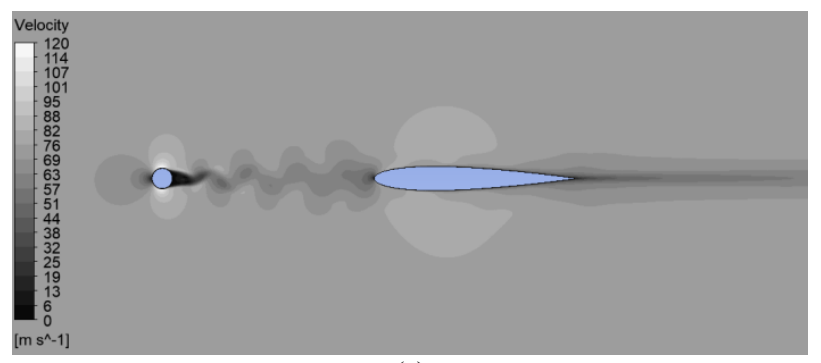

(a)

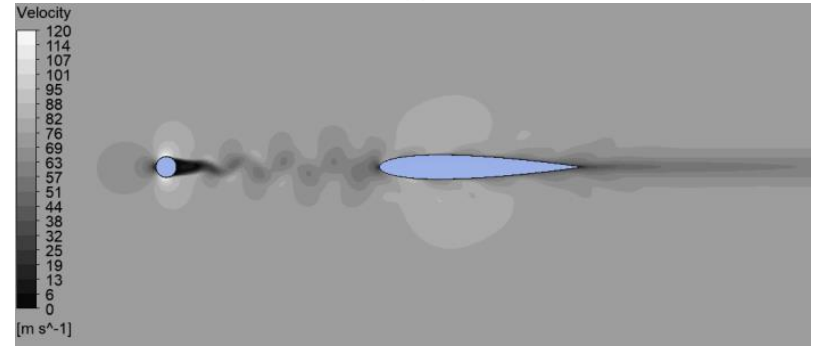

(b)

Figure 7. Velocity contours obtained for rod-airfoil configuration with (a) S-A turbulence model (b) k- $\omega$ SST turbulence model

\section{Aeroacoustic Results}

The far-field noise predictions are performed by using the FW-H approach for RANS and LES. In Figure 8, a comparison of acoustic far-field is shown for three numerical methods and experimental study [6]. Acoustic measurements are performed 18.5 chord length from the airfoil midpoint which is the same as the experiment. The first tonal peak is captured with the LES model, while RANS models capture the first peak in higher frequency values. To be able to compare the results better, in Table 1, the relative errors for frequency and magnitudes are given. It is seen that RANS models over predict the tonal frequencies with higher magnitudes. In the LES method, the Strouhal number is calculated as 0.194 with a relative error of $1.084 \%$.

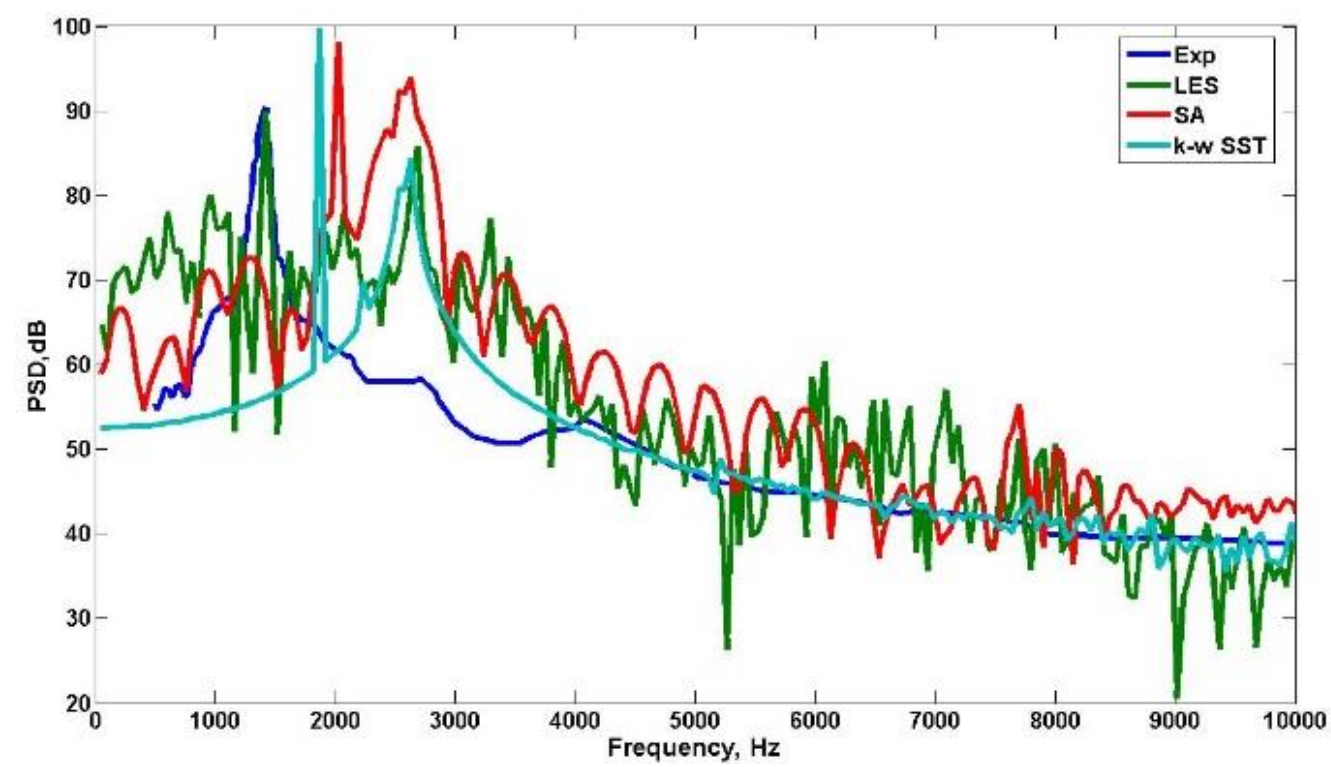

Figure 8. Comparison between experimental and numerical PSD- frequency for rod-airfoil configuration 
Journal of Thermal Engineering, Research Article, Vol. 7, No. 2, Special Issue 13, pp. 58-70, February, 2021

Table 1. Comparison of Strouhal Number and computed magnitude for RANS and LES approaches

\begin{tabular}{|c|c|c|c|c|c|}
\hline & Frequency(Hz) & St & Magnitude(dB) & \multicolumn{2}{|c|}{ Relative Error } \\
\hline & & & & $\begin{array}{c}\text { Magnitude } \\
(\mathrm{dB})\end{array}$ & St (\%) \\
\hline Experiment & 1382 & 0.191 & 90.7 & - & - \\
\hline LES & 1397 & 0.194 & 87.5 & 3.2 & 1.085 \\
\hline k-w SST & 1770 & 0.245 & 99.7 & -9 & 28.073 \\
\hline S-A & 1910 & 0.265 & 98.1 & -7.4 & 38.205 \\
\hline
\end{tabular}

In Figure 9, power spectral density against the Strouhal number is illustrated for the rod only flow. In this flow, noise is generated by a rod with a diameter of $0.01 \mathrm{~m}$. According to the results, the $\mathrm{k}-\omega$ SST model overpredicts the PSD values, while LES gives a better approach.

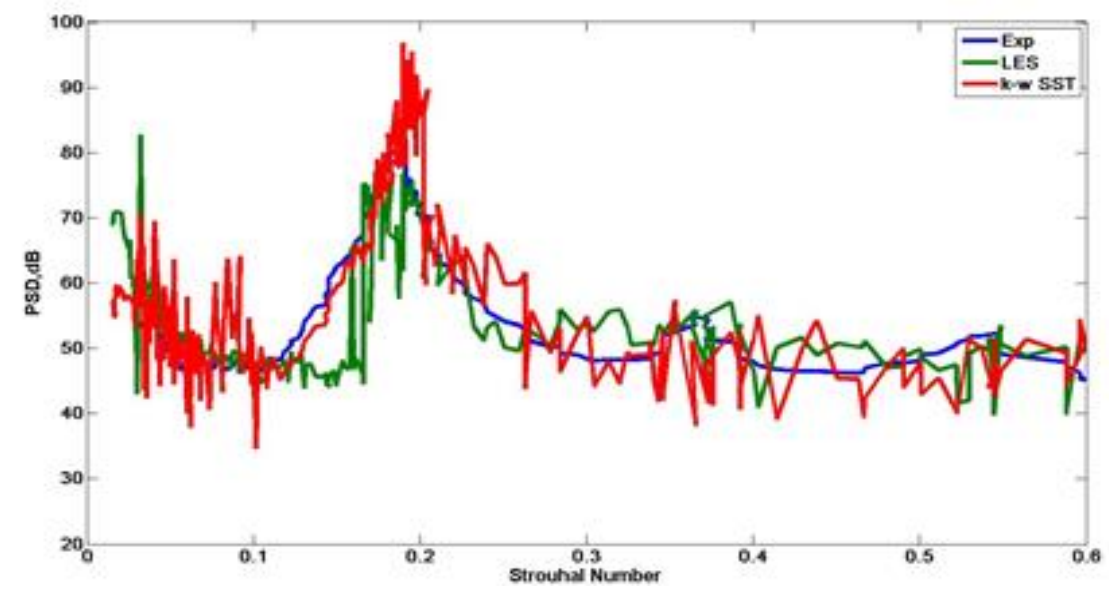

Figure 9. Variation of PSD with Strouhal number for only rod configuration

In Figure 10, the velocity contours for rod flow are shown. The periodic behaviour of the vortices is observed with a Strouhal number of 0.193. Strouhal number is calculated as 0.194 for rod-airfoil configuration. This same shedding frequency shows that spectral broadening is depended on the interaction between the incoming turbulent flow and the rod. It means that rod has a dominant effect on the shedding frequency instead of the airfoil. It is seen that the vortex shedding hit to the airfoil is the main reason for the noise generation. The rod-airfoil distance, rod diameter and flow conditions are the main parameters that have a direct effect on the St number and PSD amplitudes. In our previous research it is seen that, the vortex frequency decreases with increasing the rod diameter. To the critical diameter value, Strouhal number decreases with the rise of the diameter and after this critical diameter value, rising the rod diameter also increases the Strouhal number.

Insight of Figures 8 and 9, and Table 1, it is observed that the RANS models also predict the flow field pretty well, while the noise spectra are overestimated. Despite this, the LES model is better and yields satisfactory acoustic spectra predictions. 


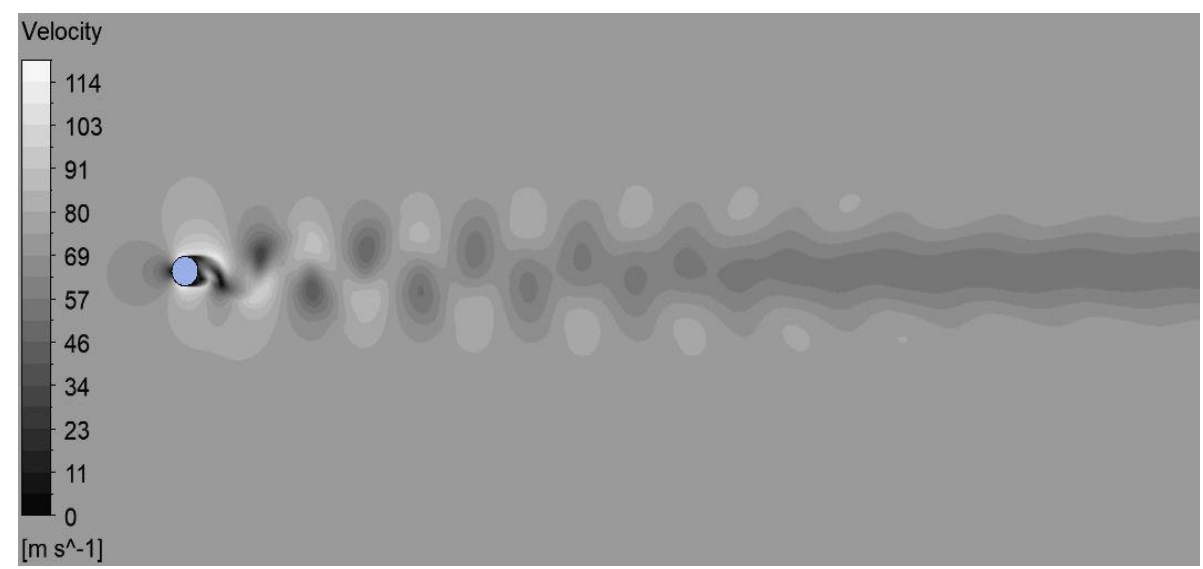

Figure 10. Velocity contour for rod flow

\section{CONCLUSION}

The rod-airfoil configuration is believed to be a benchmark for the analysis of sound generation processes in turbomachines and airfoils. In the present study, the flow structure and acoustic field are predicted by using different approaches for this problem. The main aim of the research is to examine the vortex dynamics near the rod and airfoil leading edge. Also, investigating the ability of RANS and LES approach to feature the flow in a rod-airfoil configuration is the other important part of this study. In order to demonstrate and compare the capability of LES and RANS models, acoustic and aerodynamic behavior for rod-airfoil configuration is investigated. To assess the ability of the RANS and LES approaches in the aerodynamic pattern, the velocity distributions, vorticity, and velocity contours are obtained. For comparison of acoustic spectra, spectral broadening around the shedding frequency and PSD distribution are calculated. Based on the mentioned analysis, it is observed that when the rod is located before the airfoil geometry, rod causes to the vortex street in the region between the rod and airfoil. The vortices that reach the airfoil leading edge, separates at that symmetry point and lead to the turbulent boundary layer generation. In the trailing edge of the airfoil, vortex structures form again with distortion of the vorticity field.

This flow structure is observed both with the RANS and LES methods. In the RANS method, vortices are aligned in the axis of the airfoil. On the other hand, in the experiment, irregularly settled vortices are observed due to the PIV images. This irregular vortex distribution is also observed with the LES method. With the examination of acoustic far-field results, the difference between the experiment and numerical results are obtained as 3.2, -9 and -7.4 for LES, k- $\omega$ SST and S-A models, respectively. The relative errors in numerically predicted Strouhal numbers are computed as 1.085, 28.07 and 38.2 for LES, $\mathrm{k}-\omega$ SST and S-A models, respectively. The RANS models predict the flow field well; however, they overestimate the noise spectra. LES model yields satisfactory acoustic spectra predictions. As a further study, DES and DNS models can be utilized and compared for further airfoil-turbulence interaction noise measurement.

\section{NOMENCLATURE}

d

$\mathrm{f}$

$\mathrm{k}$

$\mathrm{L}_{\mathrm{r}}$

$\mathrm{M}$

$\mathrm{P}$

$\operatorname{Re}$

PSD

$\bar{S}_{i j}$

St

$t$
Chord Length, $\mathrm{m}$

Rod Diameter, $m$

Frequency, $\mathrm{sec}^{-1}$

Turbulence Kinetic Energy

Recirculation Zone Length, $\mathrm{m}$

Mach Number

Acoustic pressure fluctuation, $\mathrm{Pa}$

Reynolds number based on the rod diameter, $\mathrm{m}$

Power Spectral Density, dB

Large-Scale Strain Rate Tensor

Strouhal Number

Time, sec 


$\begin{array}{ll}\text { Tij } & \text { Lighthill's Stress Tensor } \\ \Delta \mathrm{t} & \text { Time step size, } \mathrm{sec} \\ \mathrm{u} & \text { Fluid Velocity, } \mathrm{m} / \mathrm{s}\end{array}$

Greek symbols

$\begin{array}{ll}\varepsilon & \text { Dissipation } \\ \rho & \text { Density of a fluid. } \mathrm{kg} / \mathrm{m}^{3} \\ \omega & \text { Specific rate of dissipation }\end{array}$

Subscripts

$d \quad$ Refers to the rod diameter

c Refers to cod diameter

$i \quad$ Component in corresponding direction

$j \quad$ Component in corresponding direction

$0 \quad$ Refers to freestream

\section{REFERENCES}

[1] Casalino D., Jacob M.C., Roger M., Prediction of Rod-Airfoil Interaction Noise Using the Ffowcs-WilliamsHawkings Analogy, AIAA Journal,2008; 41: 182-191.2003.

[2] Lighthill M.J., On sound generated aerodynamically. Proc Roy Soc Lond Ser A, 1952, 211, 564-87.

[3] Ffowcs Williams J.E., Hawkings D.L., Sound generated by turbulence and surfaces in arbitrary motion, Phil Trans Roy Soc, 1969, A264(1151), 321-42.

[4] Boudet J., Casalino D., Jacob M.C., Ferrand P., Unsteady RANS Computations of the Flow Past an Airfoil in the Wake of a Rod, ASME 2002 Joint U.S.-European Fluids Engineering Division Conference

[5] Berland J., Lafon P., Crouzet F., Daude F., Bailly C., Numerical Insight into Sound Sources of a Rod-Airfoil Flow Configuration Using Direct Noise Calculation, 2010, 16th AIAA/CEAS Aeroacoustics Conference,31st AIAA Aeroacoustics Conference.

[6] Jacob M.C., Boudet J., Casalino D., Michard M., A rod-airfoil experiment as a benchmark for broadband noise modeling, Theoretical and Computational Fluid Dynamics, 2005,19, 171-196.

[7] Eltaweel, A. and Wang, M., Numerical Simulation of Broadband Noise from Airfoil-Wake Interaction, 2011, 17th AIAA/CEAS Aeroacoustics Conference, AIAA.

[8] Agrawal B.J., Sharma A., Aerodynamic Noise Prediction for a Rod-Airfoil Configuration using Large Eddy Simulations, 2014,20th AIAA/CEAS Aeroacoustics Conference.

[9] Greschner B, Thiele F, Casalino D, Jacob MC., Influence of turbulence modeling on the broadband noise simulation for complex flows, AIAA, 2004,Paper No. 2004-2902.

[10] Spalart, PRaA, S1 Allmaras., A one-equation turbulence model for aerodynamic flows, In 30th aerospace sciences meeting and exhibit.

[11] Samion, S.R.L., Ali M.S.M., Aerodynamic noise measurement in anechoic wind tunnel of rod-airfoil with leading edge serrations, Journal of Advanced Research in Fluid Mechanics and Thermal Sciences, 2018, 47 (1) 97-107.

[12] Galdeona S., Barre S., Reau N., Noise Radiated by rod-airfoil configuration using DES and the FfowcsWilliams\&Hawkings, 2010, In 16th AIAA/CEAS Aeroacoustics Conference.

[13] Caraeni, M., Dai, Y:, Caraeni, D., Acoustic Investigation of Rod Airfoil Configuration with DES and FWH, 37th AIAA Fluid Dynamics Conference and Exhibit, 25-28 June 2007, Miami, FL

[14] Michel, U., Eschriccht, D., Greschner, B., Knacke, T., Mockett, C., Thiele, F., Advanced DES Methods and Their Application to Aeroacoustics, Progress in Hybrid RANS-LES Modelling. Notes on Numerical Fluid Mechanics and Multidisciplinary Design, vol 111.

[15] Greschner,B., Peth, S., Moon, Y.J., Seo, J.H., Jacob, M.C., Thiele, F., Three- Dimensional Predictions of the Rod Wake-Airfoil Interaction Noise By Hybrid Methods, 14th International Conference on Sound \& Vibration, ICSV14, Cairns, Australia, 2007.

[16] Laouira, H., Mubarek-Oudina, F., Hussein, A.K., Kolsi, L., Merah, A., Younis, O., Heat transfer inside a horizontal channel withan open trapezoidal enclosure subjected to aheat source of different lengths, Heat Transfer, Wiley, vol (49), pp:406-423, 2020

[17] Zhiyin Y., Large-Eddy Simulation: Past, Present and the Future, Chinese Journal of Aeronautics, 2014,28, $11-24$.

[18] Ansys Fluent Manual, Fluent Inc., 2006. 
Journal of Thermal Engineering, Research Article, Vol. 7, No. 2, Special Issue 13, pp. 58-70, February, 2021

[19] Gumus B., Wind Noise Prediction of a car Model Through Solutions of Navier Stokes and Ffowcs Williams\&Hawkings Equations, MSc Thesis, Middle East Technical University, Ankara, TR.2017.

[20] Howell JR, Menguc MP, Siegel R. Thermal Radiation Heat Transfer. CRC Press; 2015.

[21] Fan L-S, Zhu C. Principles of Gas-Solid Flows Cambridge Series in Chemical Engineering. United Kingdom: Cambridge University Press; 2005.

[22] Otanicar T, Taylor RA, Phelan PE, Prasher R. Impact of size and scattering mode on the optimal solar absorbing nanofluid. Proc. ASME 3rd Int. Conf. Energy Sustain. 2009, ES2009, vol. 1, 2009, p. 791-6. https://doi.org/10.1115/ES2009-90066.

[23] Kocak, E., Aylı, E., Türkoğlu, H., Kanat Profili-Silindir Konfigürasyonunun Aerodinamik ve Aeroakustik Performansının Sayısal Analizi, 2019,14. Ulusal Tesisat Mühendisliği Kongresi, İzmir, Turkey. 